\title{
Integrating drug concentrations and minimum inhibitory concentrations with Bayesian-dose optimisation for multidrug- resistant tuberculosis
}

\author{
To the Editor:
}

The problems of multidrug-resistant (MDR) tuberculosis (TB), extensively drug-resistant (XDR) TB, and even "totally drug-resistant" TB (a term that is still debated), have exposed two important clinical issues [1, 2]. The first issue involves figuring out a way to prevent these difficult-to-treat diseases. The second issue is treating patients with drug-resistant TB [3]. In the meantime even the current susceptibility breakpoints have been challenged as being too high, so that potentially more drug-resistant TB exists than anticipated [4]. This latter aspect suggests that we need a better idea of the minimum inhibitory concentrations (MICs) to both first-line drugs and second-line drugs. Here we propose the use of MICs and drug concentrations to calculate pharmacokinetic/pharmacodynamics (PK/PD) target exposures and then to use Bayesian-feedback dosing as an integrated solution that could improve treatment outcomes.

$\mathrm{PK} / \mathrm{PD}$ relationships are deterministic, which means that the PK/PD parameters and exposures associated with certain degrees of microbial responses can be identified in preclinical models and will apply to humans. These indices include specific $24 \mathrm{~h}$ area under the concentration-time curve (AUC) to MIC ratios, such as a ratio 209 associated with optimal efficacy of pyrazinamide at the site of pulmonary infection [5]. AUC/MIC ratios of 106 (total drug) have also been identified as optimal for moxifloxacin [6]. Given the deterministic nature of the relationship, it means that these are rational therapeutic targets for optimal dosing.

Unlike PK/PD parameters, both human pharmacokinetics and MICs to Mycobacterium tuberculosis are highly variable, making it difficult to predict the actual AUCs and MICs in each patient a priori. Given that these are nondeterministic systems, it is not useful to assume that the particular MDR-TB patient in front of the practitioner has "average" drug exposures simply because they received the recommended dose or has M. tuberculosis with a certain typical MIC. Such pharmacokinetic and MIC variability drives efficacy in patients [7]. We propose that MICs to all the drugs used for treatment of the MDR-TB should be identified for each M. tuberculosis isolate, in order to capture that variability. Moreover, concentrations for drugs such as pyrazinamide, moxifloxacin, clarithromycin, linezolid, which are used to treat MDR-TB and XDR-TB [8, 9], should be measured at several time-points, in a dosing interval for each patient during the first few weeks, thereby, identifying the exact pharmacokinetic parameters, including AUC and peak concentration, so the actual AUC/MIC and peak/MIC ratios can be calculated for each patient. These exposures can then be used to estimate those in the lung, based on penetration ratios, such as those derived by AKKERMAN et al. [3]. If the AUC/MIC ratio in the lung is less than the one known to be optimal, then the dose the patient was on, as well as the pharmacokinetic parameters achieved in that patient for that drug, can be used as Bayesian priors to calculate a new dose that would achieve the optimal exposures. The drug concentrations can then be checked again after this establishing the new dose to verify that optimal AUC/MIC ratios have been achieved. The process can then be repeated if the target was not achieved. Hence, no more guessing!

One of the drawbacks of second-line drugs is their considerable toxicity profiles, especially given the duration of therapy for MDR-TB and XDR-TB. Bayesian-dose optimisation is a perfect foil for this problem for drugs whose toxicity is concentration driven. The relationship between toxicity and several second-line drugs such as linezolid and moxifloxacin, for example, have been excellently elucidated $[9,10]$, as has that of pyrazinamide [7]. When doses are adjusted after measurement of drug concentrations, the concentrations associated with increased toxicity are taken into consideration. A dose can be reduced if it already exceeds the optimal AUC/MIC but is associated with toxicity, so that the new one achieves concentrations below those that are toxic but above those associated with optimal efficacy. Thus, efficacy for treatment of MDR-TB can be further optimised while the toxicity, which is one of the limitations of second-line drugs, is reduced.

The main drawbacks to the approach we propose are the complexity and the cost, especially in low and middle income countries. However, we have practised medicine in such countries, and seen multiple anaesthetic regimens titrated by nurse anaesthetists and individualised doses delivered during surgery, even 
in rural hospitals. Thus we are confident that our proposals can be implemented in the more advanced centres in the places that have been specifically set up for the treatment of MDR-TB. There can be nothing special about achieving the same individualisation in the treatment for MDR-TB, as long as clinicians in those places are trained for the Bayesian-dose optimisation process. Indeed, we consider this approach less costly than losing patients to MDR and XDR-TB, and the cost of secondary cases.

O

@ERSpublications

Bayesian-dose optimisation for better and cost effective treatment of multi- and extremely drug resistant tuberculosis http://ow.ly/pN6KC

Shashikant Srivastava and Tawanda Gumbo

Office of Global Health, University of Texas Southwestern Medical Center, Dallas, TX, USA.

Correspondence: S. Srivastava, Office of Global Health, 5323 Harry Hines Blvd, University of Texas Southwestern Medical Center, Dallas, Texas -75390-8507, TX, USA. E-mail: shashi.kant@utsouthwestern.edu

Received: May 112013 | Accepted: May 142013

Conflict of interest: None declared.

\title{
References
}

1 Migliori GB, Sotgiu G, Gandhi NR, et al. Drug resistance beyond extensively drug-resistant tuberculosis: individual patient data meta-analysis. Eur Respir J 2013; 42: 169-179.

2 Srivastava S, Peloquin CASG, Migliori GB. Therapeutic drug management: is it the future of MDR-TB treatment? Eur Respir J 2013; 42: 1449-1453.

3 Akkerman AO, Van Altena R, Klinkenberg T, et al. Drug concentration in lung tissue in multidrug resistant tuberculosis. Eur Respir J 2013; 42: 1750-1752.

4 Gumbo T. New susceptibility breakpoints for first-line antituberculosis drugs based on antimicrobial pharmacokinetic/pharmacodynamic science and population pharmacokinetic variability. Antimicrob Agents Chemother 2010; 54: 1484-1491.

5 Gumbo T, Siyambalapitiyage Dona CSW, Leef R. Pharmacokinetics-pharmacodynamics of pyrazinamide in a novel in vitro model of tuberculosis for sterilizing effect: a paradigm for faster assessment of new antituberculosis drugs. Antimicrob Agents Chemother 2009; 53: 3197-3204.

6 Gumbo T, Louie A, Deziel MR, et al. Selection of a moxifloxacin dose that suppresses drug resistance in Mycobacterium tuberculosis, by use of an in vitro pharmacodynamic infection model and mathematical modeling. J Infect Dis 2004; 190: 1642-1651.

7 Srivastava S, Pasipanodya JG, Meek C, et al. Multidrug-resistant tuberculosis not due to noncompliance but to between-patient pharmacokinetic variability. J Infect Dis 2011; 204: 1951-1959.

8 Bolhuis MS, Altena R, van Soolingen D, et al. Clarithromycin increases linezolid exposure in multidrug-resistant tuberculosis patients. Eur Respir J 2013; 42: 1614-1621.

9 Sotgiu G, Centis R, D'Ambrosio L, et al. Efficacy, safety and tolerability of linezolid containing regimens in treating MDR-TB and XDR-TB: systematic review and meta-analysis. Eur Respir J 2012; 40: 1430-1442.

10 Haverkamp W, Kruesmann F, Fritsch A, et al. Update on the cardiac safety of moxifloxacin. Curr Drug Saf 2012; 7: $149-163$.

\section{MicroRNAs and pulmonary hypertension}

\author{
To the Editor:
}

MicroRNAs have emerged as important posttranscriptional regulators of gene transcription. The interesting review by RUPANI et al. [1] on microRNAs in respiratory diseases is, thus, accurately timed. We use this opportunity to additionally mention the role of microRNAs in pulmonary hypertension, which has been investigated both in experimental models and in human disease and, as recently reviewed in the European Respiratory Journal [2], might be of pathogenetic relevance for pulmonary hypertension. Caruso et al. [3], for example, described alterations in the expression of dicer, which is one of the most important microRNA processing enzymes, probably explaining the reduced expression levels of several microRNAs in patients with pulmonary hypertension. Some of these, such as miR-150 [4], have been described as independent predictors for an adverse outcome. Others, including miR-204 [5] have been linked to important signalling pathways in pulmonary arterial smooth muscle cells. Finally, our own work, has identified the microRNA cluster 17/92 as directly targeting the bone morphogenetic protein receptor type II [6], which, as shown by successful inhibition by antagomirs in vivo $[7,8]$, could be a causative therapeutic approach for the vascular remodelling of pulmonary arteries. 